\title{
FINITE ELEMENT ANALYSES OF TURBINE DISC WITH SURFACE CRACKS
}

\author{
Bhanu Murthy Soppari ${ }^{1}$ \\ ${ }^{1}$ Assistant professor, Mechanical Engineering Department, VNR VJIET, Telangana, India
}

\begin{abstract}
In Aircrafts, Gas turbine discs are critical engine components, which must endure substantial mechanical and thermal loading, such air facts will be in trouble if an induced cracks develop and may gradually affect the whole engine function and leads to failure. In order to overcome the problem turbine life span has to be predicted using the approximate methods of evaluation (Ansys workbench software). There are a number of investigations devoted to both failure analyses and life assessment of turbine discs. Therefore, it is important to get approximate life of the turbine disc by simulating the fatigue damage of crack growth rate, and are essential in determining either the likely failure modes or the component replacement intervals to prevent failures under normal operating conditions.
\end{abstract}

Keywords: Gas turbine disc, Solid works, Ansys workbench, Crack growth, Failure modes, Applications. - ***.

\section{INTRODUCTION}

The gas turbine is a turbine which uses very highly pressurized gas as the charge for working medium and its component of a power plant which works on the principle of Brayton cycle, power plant mainly consists of an upstream rotating compressor which compresses the incoming ambient air to high pressure. Compressed air is sent to the combustion where it is mixed with fuel and gets combusted to a high temperature. As a result, combustion takes place and hot gases with high energy content are generated. These hot gases are then transferred to the turbine where expansion takes place and the heat energy gets converted to rotary motion of the turbine shaft or mechanical energy. This turbine is usually coupled with a generator which then converts this mechanical energy to electrical output. The blades of a turbine play a vital role in determining the life and overall efficiency of a gas turbine. Upon impingement by high pressure gases these blades rotate and produce mechanical work.

\subsection{Modal Analysis}

To predict residual life assessment of turbine disc in a military aircraft engine is susceptible to fatigue cracking failure where flaws formed in the original manufacturing process [1]. These flaws were not considered in the original life assessment, it is important to predict the residual life of gas turbine discs and to predict the safe inspection intervals in order to prevent possible failures during service [2].

\section{PROBLEM STATEMENT}

Studying fracture analyses of turbine disc with Induced cracks of different sizes to determine the safe inspection intervals in order to prevent possible failure during service of structural steel material turbine disc in Ansys

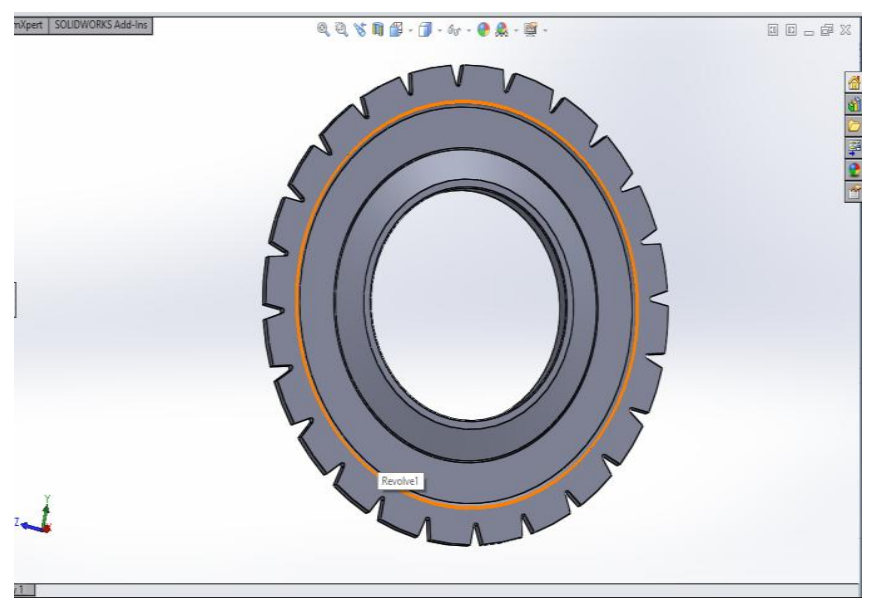

Fig -1: Turbine disc

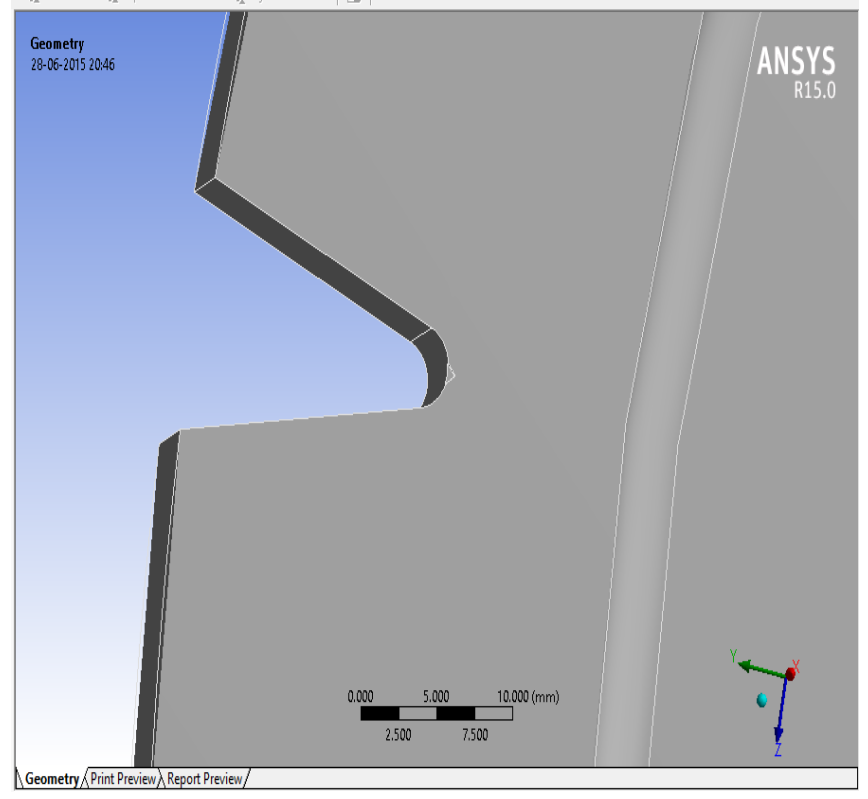

Fig -2: Turbine disc with initial crack 


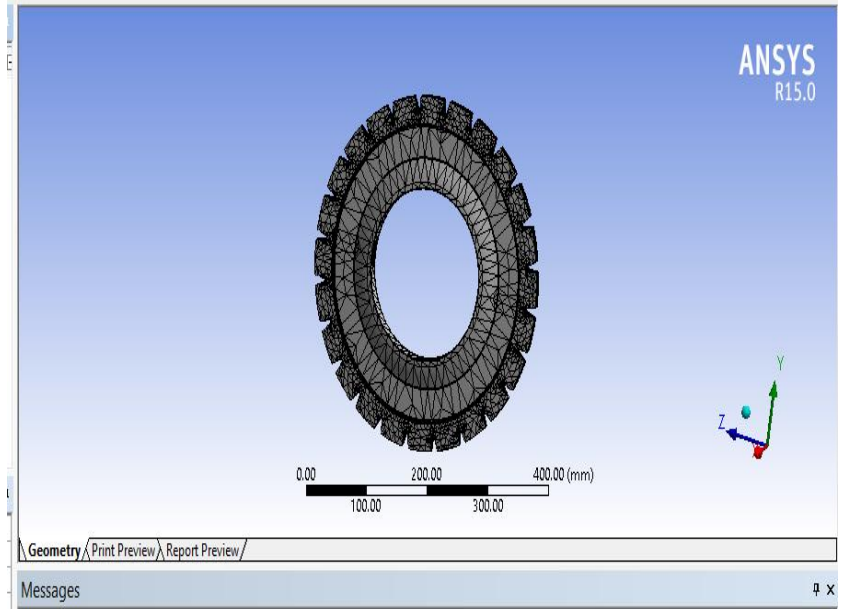

Fig -3: Turbine disc with initial crack after meshing

Table -1: Details of turbine material

\begin{tabular}{|l|l|l|l|}
\hline Material & $\begin{array}{l}\text { Density } \\
(\mathrm{kg} / \mathrm{m3})\end{array}$ & $\begin{array}{l}\text { Modulus of } \\
\text { Elasticity (GPa) }\end{array}$ & $\begin{array}{l}\text { Poisson's } \\
\text { Ratio }\end{array}$ \\
\hline $\begin{array}{l}\text { Structural } \\
\text { steel }\end{array}$ & $\mathbf{7 8 5 0}$ & $\mathbf{2 0 0}$ & $\mathbf{0 . 3 3}$ \\
\hline
\end{tabular}

\section{PERFORMING FATIGUE ANALYSIS}

Analysis is performed on the turbine disc and estimate the residual life of turbine disc:-

Table -2: Alternating stress

\begin{tabular}{|l|l|}
\hline Alternating Stress MPa & Cycles \\
\hline 3999 & 10 \\
\hline 2827 & 20 \\
\hline 1896 & 50 \\
\hline 1413 & 100 \\
\hline 1069 & 200 \\
\hline 441 & 2000 \\
\hline 262 & 10000 \\
\hline 214 & 20000 \\
\hline 138 & $1 . e+005$ \\
\hline 114 & $2 . e+005$ \\
\hline 86.2 & $1 . e+006$ \\
\hline
\end{tabular}
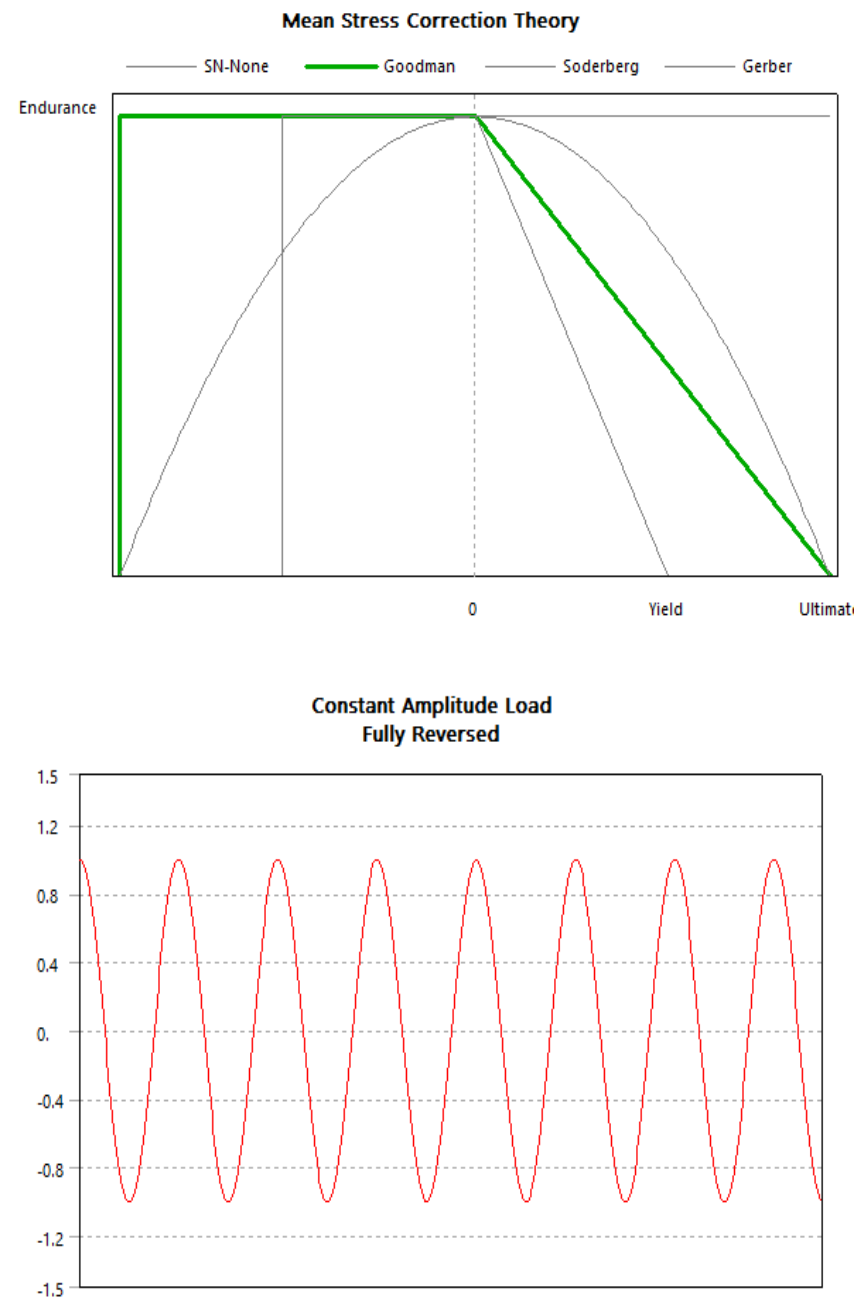

\subsection{Case 1}

Considering the turbine disc with $0.5 \mathrm{~mm} * 0.5 \mathrm{~mm}$ size crack and performed the analyses by using the rotational velocity of $1492 \mathrm{rad} / \mathrm{s}$ and the values as follows:-

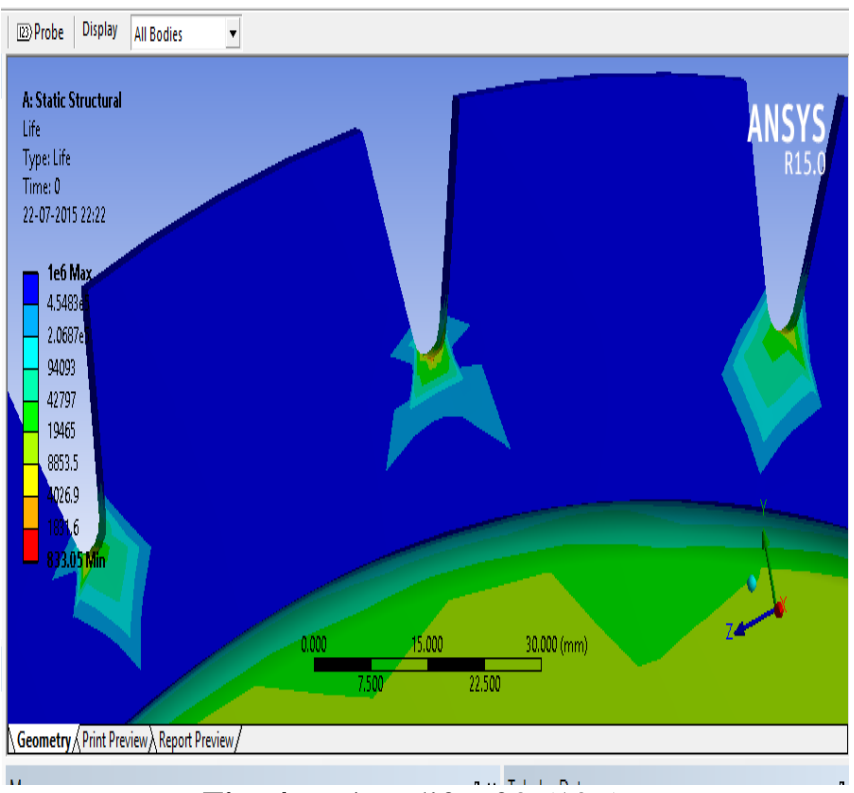

Fig -4: Fatigue life of $0.5 * 0.5 \mathrm{~mm}$ 
Table -3: Minimum life cycle

\begin{tabular}{|l|l|l|}
\hline Type & Life & Safety Factor \\
\hline Design Life & & $1 . e+009$ cycles \\
\hline Minimum & 902.41 cycles & 0.14393 \\
\hline
\end{tabular}

\subsection{Case 2}

Considering the turbine disc with $1 \mathrm{~mm} * 1 \mathrm{~mm}$ size crack and performed and the values as follows

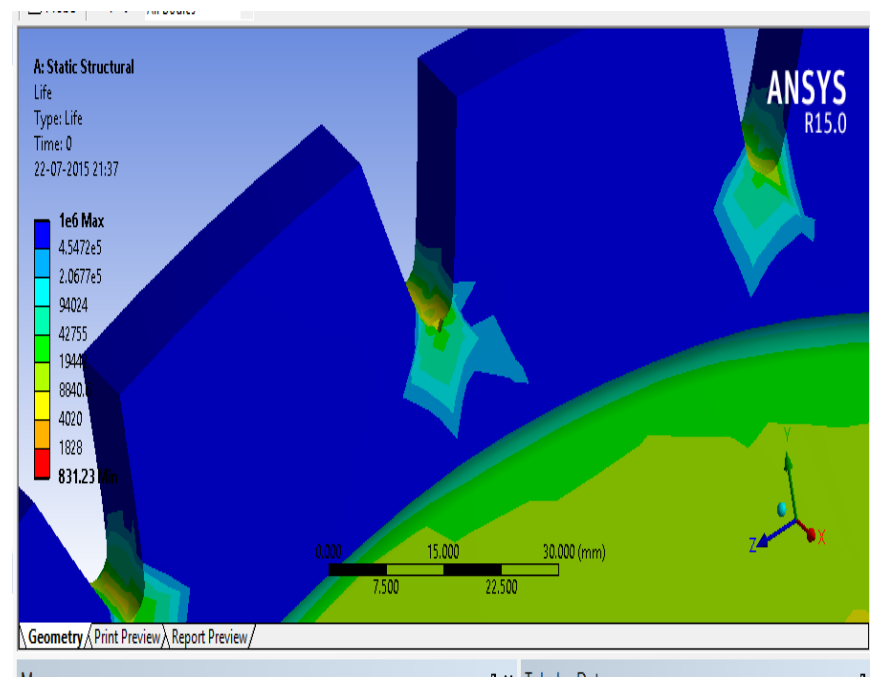

Fig -5: Fatigue life of $1 * 1 \mathrm{~mm}$

Table -4: Minimum life cycle

\begin{tabular}{|l|l|l|}
\hline Type & Life & Safety Factor \\
\hline Design Life & & 1. e+009 cycles \\
\hline Minimum & 831.23 cycles & 0.13946 \\
\hline
\end{tabular}

\subsection{Case 3}

Considering the turbine disc with $1.5 * 1.5 \mathrm{~mm}$ size crack and performed and the values as follows

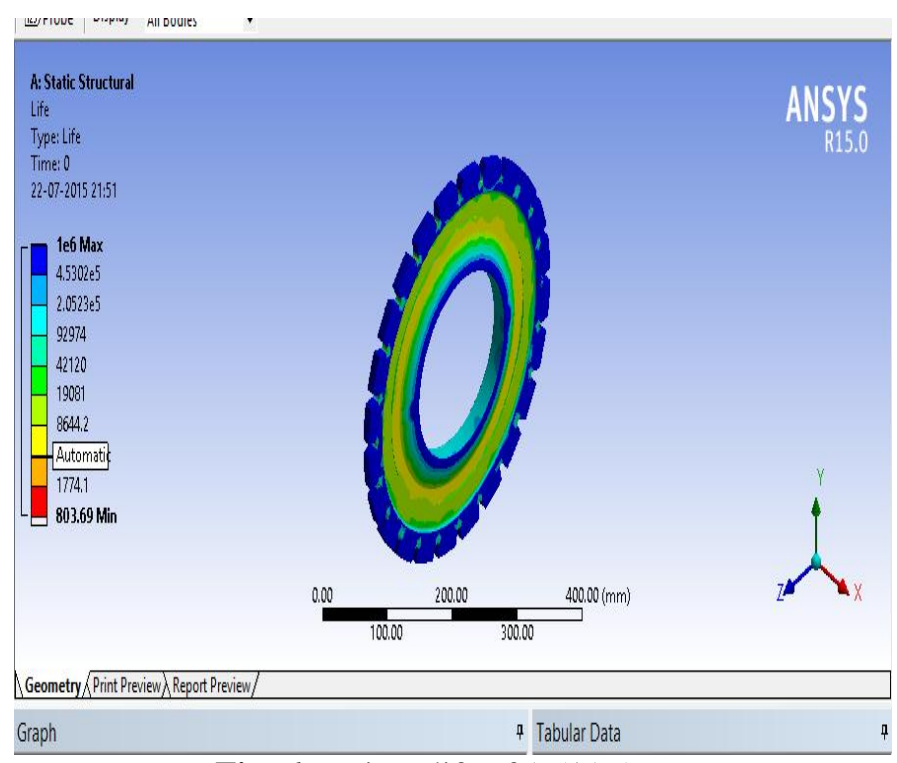

Fig -6: Fatigue life of $1.5 * 1.5 \mathrm{~mm}$
Table -5: Minimum life cycle

\begin{tabular}{|l|l|l|}
\hline Type & Life & Safety Factor \\
\hline Design Life & & $1 . e+009$ cycles \\
\hline Minimum & 803.69 cycles & 0.13766 \\
\hline
\end{tabular}

\section{RESULTS}

The results of turbine disc fatigue life of different crack sizes with single induced crack.

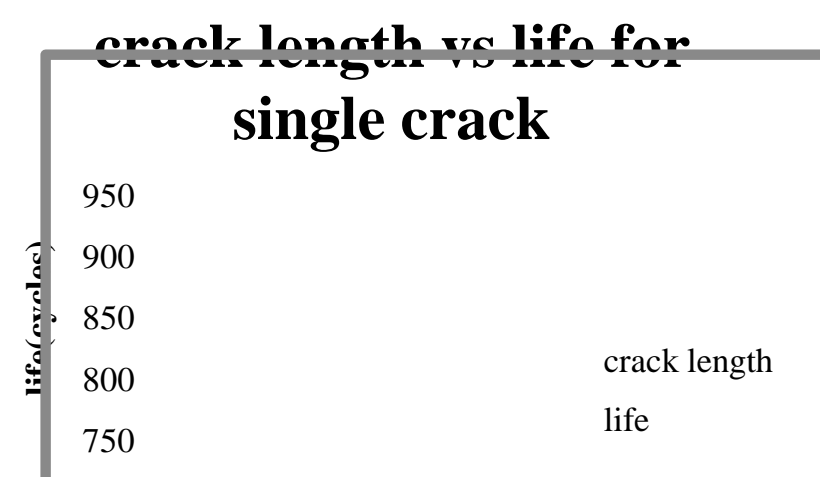

$\begin{array}{lll}0.5 & 1 & 1.5\end{array}$

crack lengths(mm)

Chart -1: Crack length Vs life

\section{CONCLUSION}

It is concluded that the residual life for the disc is being changing with respective to the size of the crack induced in the turbine disc as the size of the crack increases the life of the turbine blade decreases and stress is drastically being increased with the working environment.

\section{REFERENCES}

[1]. Harris J.Engine components retirement for cause.AFMALTR-874069; 1987.

[2]. Z huang WZ .Prediction of crack growth from bolt holes in disc a.IntJ Fatigue 2000; 22:241-50.

[3]. Hou J, Slater S ,Gold smith N, WicksBJ. Fatigue Crack Growth Prediction In Turbine Fan Disc .Symposium 2000 on Structural Integrity and Fracture on 29-30 June, 2000 at University of Technology, Sydney Australia.

[4]. HouJ, DubkeJ, BarlowK, SlaterS, HarrisL, CalcuttawalaS, BeresW.3Dcrackgrowth analysis and its correlation with experiments for critical turbine components under an international collaborative program. GT2008-50648. Proceedings of ASME Turbo Expo 2008. Berlin (Germany); June9-13;2008.

[5]. MeguidSA,KanthPS,CzekanskiA.Finite element analysis of fir tree region in turbine disc. Finite Element Anal 2000; 35:305-17. 